\title{
Antimicrobial Activities of Extracts of Some Species of Mangrove Plants and a New Compound Isolated Towards some Selected Strains
}

\author{
KARNATI. RAJESWARI', T.BHASKARA RAO', \\ G.V.R.SHARMA ${ }^{2}$ and R.MURALI KRISHNA ${ }^{3}$
}

${ }^{1}$ Department of Chemistry, K L University, Guntur, Andhra Pradesh -522502, India. 2Department of Chemistry, Gitam University, Rushikonda, Visakhapattnam -530 003, India. ${ }^{3}$ School of Chemistry, Andhra University, Visakhapatnam -530 003, India.

*Corresponding author E-mail: krajeswari@kluniversity.in

http://dx.doi.org/10.13005/ojc/330256

(Received: November 25, 2016; Accepted: February 12, 2017)

\begin{abstract}
The bio-materials of four marine mangrove medicinal plants viz., Aegiceras Corniculatum (AGC), Excoecariaagallocha (EA), Rhizophoramucronata (RM) and Xylocarpusgranatum (XG) are extracted with methanol and hexane. These extracts are submitted to the antibacterial activity towards the strains:Bacillus puvuilis, Bacillus subtilis, Bacillus coagulans, Staphylococcus aureus, Bacillus licheniformis, Corynebacterium diphtheria, Klebsiella pneumonia, Pseudomonas aeruginosa, Shigellaflexneri, Sphingomonaspaucimobilis, Escherichia coli and Vibrio choleraadopting Agarwell diffusion method. It is found that a new Flavone Compound isolated from hexane extract of EAis effective towards Bacillus puvuilis, Bacillus subtilis, Bacillus coagulans, Staphylococcus aureus, Bacillus licheniformis, Corynebacterium diphtheria, Klebsiella pneumonia, Pseudomonas aeruginosa, Shigellaflexneri, Sphingomonaspaucimobilis, Escherichia coli and Vibrio cholera strains while RM MeOH extract is effective towards the strains Bacillus puvuilis, Bacillus subtilis, Bacillus coagulans, Staphylococcus aureus, Bacillus licheniformis, Corynebacterium diphtheria, Klebsiella pneumonia, Pseudomonas aeruginosa, Shigellaflexneri, Sphingomonaspaucimobilis, Escherichia coli and Vibrio cholera. The XG MeOH extract is found to be effective towards the strains Bacillus puvuilis, Bacillus subtilis, Bacillus coagulans, Staphylococcus aureus, Bacillus licheniformis, Corynebacterium diphtheria, Klebsiella pneumonia, Pseudomonas aeruginosa, Shigellaflexneri, Sphingomonaspaucimobilis, Escherichia coli and Vibrio cholera strains while AGC MeOH extract is found to be effective towards the strainsBacillus puvuilis, Bacillus subtilis, Bacillus coagulans, Staphylococcus aureus, Bacillus licheniformis, Corynebacterium diphtheria, Klebsiella pneumonia, Pseudomonas aeruginosa, Shigellaflexneri, Sphingomonaspaucimobilis, Escherichia coli and Vibrio cholera. The order of effectiveness is found to be: EA Hexane > RM MeOH>XG MeOH>AG MeOH. Finally a new flavone compound is found to be more effective than the extracts.
\end{abstract}

Keywords: Mangrove plants, extracts, flavone compound, antimicrobial activity on different strains. 


\section{INTRODUCTION}

The recent investigations are concentrating on the bio-screening of natural products have revived due to the paucity of safe antimicrobial drugs, anti-reverse transcriptase, anti-HIV and the perilous upsurge of new and re-emerging infectious diseases ${ }^{1,2,3}$. The antibiotics from natural sources are efficacious, biodegradable, less toxic and cost effective and therefore, it could supplement

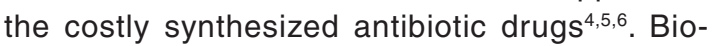
potentiality of mangrove vegetal makes them as a reserved for the development of pharmaceuticals, fish and animal feed additives, agrichemicals and natural pigments ${ }^{7,8,9}$. The mangrove preparations used successfully in the hospitalization of infectious diseases and aliments are envisaged to possess antimicrobial potency ${ }^{10,11,12}$.

In the present investigation, the different biological parts of four mangrove species namely AegicerasCorniculatum, Excoecariaagallocha, Rhizophoramucronata and Xylocarpusgranatumhave been extracted with different solvents like hexane and methanol. These extractes have been screened for antimicrobial activity towards the strains Bacillus puvuilis, Bacillus subtilis, Bacillus coagulans, Staphylococcus aureus, Bacillus licheniformis, Corynebacterium diphtheria, Klebsiella pneumonia, Pseudomonas aeruginosa, Shigellaflexneri, Sphingomonaspaucimobilis, Escherichia coli and Vibrio cholera and found to be results are encouraging hence they are presented comprehensively in this article.

\section{MATERIALS and METHODS}

\section{Collection of Mangrove Medicinal plants}

The different species of mangrove plants viz., Excoecariaagallocha and Xylocarpusgranatum, were collected from corangi Mangrove forest near Bhiravapalem in Godavary Estuary ( Latitude 160

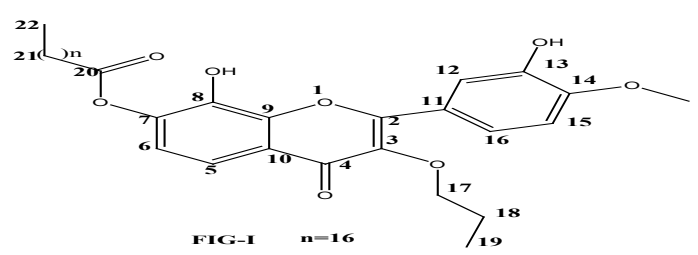

8-hydroxy-2(3-hydroxy-4-methoxy phenyl) 4-oxo-3-propoxy-4H-chromen-7yl-propionate $15^{\prime} \mathrm{N}$ and Longitude $82015^{\prime} \mathrm{E}$ ) and further , Aegiceras Corniculatum and Rhizophora Mucronata ( Latitude 80 99' N and Longitude 760 87' E) were collected from Kollam mangrove forest near Krishnapatnam Port, Nellore.

\section{Plant preparation and extraction}

The fresh plants were washed under running tap water and dried in a warm room for 2 to $6 \mathrm{~d}$. The samples were grinded into fine powder and extracted with $n$-hexane and methanol successively to get $n$-hexane and methanol extracts. Then, all the crude extracts were kept at -20 ! until further use. The flavone compound getting By using column chromatography over a column of silica gel (Acme brand, 100-200 mesh, and $450 \mathrm{~g}$ ) using solvents of increasing polarity from $n$-hexane through EtoAc. In all 200 Fractions (500 ml) were collected. The fractions displaying similar spots in TLC were combined and the residues from therein were subjected to re-chromatography over silica gel column to yield one pure compound Fig. ${ }^{13}$ In the form of an off-white solid.

\section{preparation of a sample}

A sample of $100 \mathrm{mg}$ from each extract and compound was dissolved in $1 \mathrm{~mL}$ DMSO. The extract and compound was then sterilized by filtration through sterile syringe filter $(0.2 \mu \mathrm{m}$ pore). Finally the filtered extract and compound was stored as aliquots until it was used.

\section{Microbial strains}

Bacillus puvuilis, Bacillus subtilis, Bacillus coagulans, Staphylococcus aureus, Bacillus licheniformis, Corynebacterium diphtheria, Klebsiella pneumonia, Pseudomonas aeruginosa, Shigellaflexneri, Sphingomonaspaucimobilis, Escherichia coli and Vibrio cholera.

\section{Agar Ditch diffusion method}

The agar disc diffusion method was employed for the determination of antimicrobial activities of the extracts according to Qarallehet al ${ }^{14}$ some modification. Briefly, inoculum containing $120^{\circ}$ (15 lb/in2)was spread on Nutrient agar Medium with the respective bacterial strains of bacteria and medium potato dextrose agar for fungus strains. Testing sterile forceps, the sterile filter papers $(6 \mathrm{~mm}$ diameter) containing the crude extracts (1 or $1.5 \mathrm{mg}$ ), 
standard antibiotics (30 $\mu \mathrm{g}$ of chloramphenicol or 100 $\mu \mathrm{g}$ of amphotericin B) or negative control (DMSO) were laid down on the coverage of inoculated agar plate. The plates were incubated at $37 \pm 2$ ! for $24 \mathrm{~h}$ for the bacteria and at room temperature $28 \pm 2$ ! for $12 \mathrm{~h}$ for yeasts strains. Each sample was tested in duplicate and the zone of inhibition was measured as 50 micro liters diameter.

\section{Screening for Antimicrobial Activity}

The antimicrobial activity was carried out by the employing $24 \mathrm{~h}$ young cultures with the given compounds by using Agar-well diffusion method. The medium was sterilized by autoclaving at $120^{\circ} \mathrm{C}$ (15 lb/in2). About $20 \mathrm{ml}$ of the medium (Nutrient Agar Medium) with the respective bacterial strains of bacteria and medium (Potato Dextrose Agar) for Fungal strains were transferred aseptically into each sterilized petri Plate. The plates were left at room temperature for solidification. Each plate is made 5 wells with equal distance with of $6 \mathrm{~mm}$ sterile borer. The test compounds were freshly reconstituted with suitable solvents (DMSO) and tested at various concentrations. The samples and the control along with standard (Ciprofloxacin) were places in 6-mm diameter well. In Antimicrobial assays plates were incubated at $28 \pm 20$ c for fungi about $24 \mathrm{~h}$ and $37 \pm 20 \mathrm{C}$

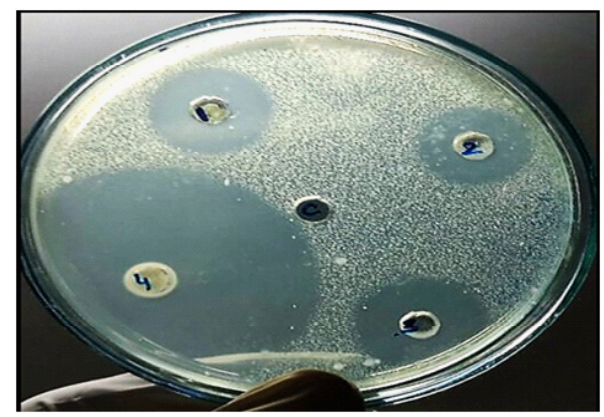

Fig. 2: Mangrove plants extracts and a new flavone compound activity on some selected strains for bacteria $12 \mathrm{~h}$. Standard with $5 \mu \mathrm{g} / \mathrm{ml}$ used as a positive control for antibacterial activity. Activity diameter of the zone of inhibition was measured using Himedia antibiotic zone scale. Observations and results were represented in Table 2.

\section{RESULTS and DISCUSSION}

The Agar well diffusion method which belongs to Gram positive \& Gram negative Bacteria's of different plant extracts and flavone compound towards different strains have been presented in Table 2. The following observations are significant: of all the extracts and compound tested, AGC, EA, $\mathrm{RM}, \mathrm{XG}$ have shown some remarkable antimicrobial behaviour.

\section{With AGC extract, the antimicrobial activity for} strains

Bacillus puvuilis, Bacillus subtilis, Bacillus coagulans, Staphylococcus aureus, Bacillus licheniformis, Corynebacterium diphtheria, Klebsiella pneumonia, Pseudomonas aeruginosa, Shigella flexneri, Sphingomonas paucimobilis, Escherichia coli and Vibrio cholera.

The Gram Positive Bacteria's are Bacillus subtilis with the values 12 and 10 respectively, Bacillus coagulans with the values 13,11 and 10 respectively. And Staphylococcus aureus with the value 7 respectively. These strains have no activity against the Gram Negative Bacteria's.

With EA flavone compound, the antimicrobial activity for strains

Bacillus puvuilis, Bacillus subtilis, Bacillus coagulans, Staphylococcus aureus, Bacillus licheniformis, Corynebacterium diphtheria, Klebsiella pneumonia, Pseudomonas aeruginosa, Shigella flexneri, Sphingomonas paucimobilis, Escherichia coli and Vibrio cholera.The Gram Positive Bacteria's

Table 1: Abbreviation of Mangrove Medicinal Plant Extracts \& Compound

\begin{tabular}{llll}
\hline Name of the Plant Species & Parts used & Extraction of solvent & Abbreviation \\
\hline AegicerasCorniculatum & Fruits & Methanol & AGC \\
Excoecariaagallocha (compound) & Roots & Hexane & EA \\
Rhizophoramucronata & Fruits & Methanol & RM \\
XylocarpusGranatum & Roots & Methanol & XG \\
\hline
\end{tabular}


Table 2: Results of Antimicrobial Assay mangrove medicinal plants

\begin{tabular}{|c|c|c|c|c|c|c|c|}
\hline \multicolumn{8}{|c|}{ Gram Positive Bacteria's } \\
\hline $\begin{array}{l}\text { S. } \\
\text { No. }\end{array}$ & $\begin{array}{l}\text { Plant } \\
\text { code }\end{array}$ & Organism/s & $500 \mathrm{mg} / \mathrm{ml}$ & $250 \mathrm{mg} / \mathrm{ml}$ & $100 \mathrm{mg} / \mathrm{ml}$ & Standard & Control \\
\hline 1 & AGC & Bacillus puvuilis & No Activity & No Activity & No Activity & $43 \mathrm{~mm}$ & No Activity \\
\hline 2 & AGC & Bacillus subtilis & $12 \mathrm{~mm}$ & $10 \mathrm{~mm}$ & No Activity & $40 \mathrm{~mm}$ & No Activity \\
\hline 3 & AGC & Bacillus coagulans & $13 \mathrm{~mm}$ & $11 \mathrm{~mm}$ & $10 \mathrm{~mm}$ & $34 \mathrm{~mm}$ & No Activity \\
\hline 4 & AGC & Staphylococcus aureus & $7 \mathrm{~mm}$ & No Activity & No Activity & $40 \mathrm{~mm}$ & No Activity \\
\hline 5 & AGC & Bacillus licheniformis & No Activity & No Activity & No Activity & $34 \mathrm{~mm}$ & No Activity \\
\hline 6 & AGC & Corynebacteriumdiphtheriae & No Activity & No Activity & No Activity & $36 \mathrm{~mm}$ & No Activity \\
\hline 7 & EA & Bacillus puvuilis & $19 \mathrm{~mm}$ & $18 \mathrm{~mm}$ & $17 \mathrm{~mm}$ & $43 \mathrm{~mm}$ & No Activity \\
\hline 8 & $\mathrm{EA}$ & Bacillus subtilis & No Activity & No Activity & No Activity & $40 \mathrm{~mm}$ & No Activity \\
\hline 9 & $E A$ & Bacillus coagulans & No Activity & No Activity & No Activity & $40 \mathrm{~mm}$ & No Activity \\
\hline 10 & $\mathrm{EA}$ & Staphylococcus aureus & $13 \mathrm{~mm}$ & $12 \mathrm{~mm}$ & $11 \mathrm{~mm}$ & $40 \mathrm{~mm}$ & No Activity \\
\hline 11 & $\mathrm{EA}$ & Bacillus licheniformis & $14 \mathrm{~mm}$ & $12 \mathrm{~mm}$ & $11 \mathrm{~mm}$ & $32 \mathrm{~mm}$ & No Activity \\
\hline 12 & EA & Corynebacteriumdiphtheriae & $11 \mathrm{~mm}$ & $10 \mathrm{~mm}$ & No Activity & $43 \mathrm{~mm}$ & No Activity \\
\hline 13 & $\mathrm{RM}$ & Bacillus puvuilis & $12 \mathrm{~mm}$ & No Activity & No Activity & $43 \mathrm{~mm}$ & No Activity \\
\hline 14 & $\mathrm{RM}$ & Bacillus subtilis & No Activity & No Activity & No Activity & $40 \mathrm{~mm}$ & No Activity \\
\hline 15 & $\mathrm{RM}$ & Bacillus coagulans & $15 \mathrm{~mm}$ & $13 \mathrm{~mm}$ & $11 \mathrm{~mm}$ & $40 \mathrm{~mm}$ & No Activity \\
\hline 16 & $\mathrm{RM}$ & Staphylococcus aureus & No Activity & No Activity & No Activity & $38 \mathrm{~mm}$ & No Activity \\
\hline 17 & $\mathrm{RM}$ & Bacillus licheniformis & No Activity & No Activity & No Activity & $28 \mathrm{~mm}$ & No Activity \\
\hline 18 & $\mathrm{RM}$ & Corynebacteriumdiphtheriae & No Activity & No Activity & No Activity & $34 \mathrm{~mm}$ & No Activity \\
\hline 19 & $X G$ & Bacillus puvuilis & No Activity & No Activity & No Activity & $43 \mathrm{~mm}$ & No Activity \\
\hline 20 & $X G$ & Bacillus subtilis & $12 \mathrm{~mm}$ & $10 \mathrm{~mm}$ & No Activity & $40 \mathrm{~mm}$ & No Activity \\
\hline 21 & $X G$ & Bacillus coagulans & $15 \mathrm{~mm}$ & $11 \mathrm{~mm}$ & No Activity & $40 \mathrm{~mm}$ & No Activity \\
\hline 22 & $X G$ & Staphylococcus aureus & $14 \mathrm{~mm}$ & $13 \mathrm{~mm}$ & $12 \mathrm{~mm}$ & $40 \mathrm{~mm}$ & No Activity \\
\hline 23 & $X G$ & Bacillus licheniformis & $11 \mathrm{~mm}$ & $10 \mathrm{~mm}$ & No Activity & $35 \mathrm{~mm}$ & No Activity \\
\hline 24 & $X G$ & Corynebacteriumdiphtheriae & No Activity & No Activity & No Activity & $34 \mathrm{~mm}$ & No Activity \\
\hline \multicolumn{8}{|c|}{ Gram Negative Bacteria's } \\
\hline $\begin{array}{l}\text { S. } \\
\text { No. }\end{array}$ & $\begin{array}{l}\text { Plant } \\
\text { code }\end{array}$ & Organism/s & $500 \mathrm{mg} / \mathrm{ml}$ & $250 \mathrm{mg} / \mathrm{ml}$ & $100 \mathrm{mg} / \mathrm{ml}$ & Standard & Control \\
\hline 1 & AGC & Klebsiellapneumoniae & No Activity & No Activity & No Activity & $32 \mathrm{~mm}$ & No Activity \\
\hline 2 & AGC & Pseudomonas aeruginosa & No Activity & No Activity & No Activity & $38 \mathrm{~mm}$ & No Activity \\
\hline 3 & AGC & Shigellaflexneri & No Activity & No Activity & No Activity & $38 \mathrm{~mm}$ & No Activity \\
\hline 4 & AGC & Sphingomonaspaucimobilis & No Activity & No Activity & No Activity & $31 \mathrm{~mm}$ & No Activity \\
\hline 5 & AGC & Escherichia coli & No Activity & No Activity & No Activity & $40 \mathrm{~mm}$ & No Activity \\
\hline 6 & AGC & Vibrio cholerae & No Activity & No Activity & No Activity & $29 \mathrm{~mm}$ & No Activity \\
\hline 7 & EA & Klebsiellapneumoniae & $11 \mathrm{~mm}$ & No Activity & No Activity & $28 \mathrm{~mm}$ & No Activity \\
\hline 8 & EA & Pseudomonas aeruginosa & $10 \mathrm{~mm}$ & No Activity & No Activity & $38 \mathrm{~mm}$ & No Activity \\
\hline 9 & EA & Shigellaflexneri & $11 \mathrm{~mm}$ & No Activity & No Activity & $32 \mathrm{~mm}$ & No Activity \\
\hline 10 & $E A$ & Sphingomonaspaucimobilis & $15 \mathrm{~mm}$ & No Activity & No Activity & $40 \mathrm{~mm}$ & No Activity \\
\hline 11 & $E A$ & Escherichia coli & No Activity & No Activity & No Activity & $40 \mathrm{~mm}$ & No Activity \\
\hline 12 & $\mathrm{EA}$ & Vibrio cholerae & $13 \mathrm{~mm}$ & $12 \mathrm{~mm}$ & $11 \mathrm{~mm}$ & $40 \mathrm{~mm}$ & No Activity \\
\hline 13 & $\mathrm{RM}$ & Klebsiellapneumoniae & $20 \mathrm{~mm}$ & $13 \mathrm{~mm}$ & $10 \mathrm{~mm}$ & $32 \mathrm{~mm}$ & No Activity \\
\hline 14 & $\mathrm{RM}$ & Pseudomonas aeruginosa & $15 \mathrm{~mm}$ & No Activity & No Activity & $40 \mathrm{~mm}$ & No Activity \\
\hline 15 & $\mathrm{RM}$ & Shigellaflexneri & $16 \mathrm{~mm}$ & $12 \mathrm{~mm}$ & No Activity & $40 \mathrm{~mm}$ & No Activity \\
\hline 16 & $\mathrm{RM}$ & Sphingomonaspaucimobilis & $19 \mathrm{~mm}$ & $13 \mathrm{~mm}$ & $11 \mathrm{~mm}$ & $30 \mathrm{~mm}$ & No Activity \\
\hline 17 & $\mathrm{RM}$ & Escherichia coli & $16 \mathrm{~mm}$ & $12 \mathrm{~mm}$ & No Activity & $40 \mathrm{~mm}$ & No Activity \\
\hline 18 & $\mathrm{RM}$ & Vibrio cholerae & $19 \mathrm{~mm}$ & $13 \mathrm{~mm}$ & $10 \mathrm{~mm}$ & $35 \mathrm{~mm}$ & No Activity \\
\hline 19 & $X G$ & Klebsiellapneumoniae & No Activity & No Activity & No Activity & $32 \mathrm{~mm}$ & No Activity \\
\hline 20 & $X G$ & Pseudomonas aeruginosa & No Activity & No Activity & No Activity & $40 \mathrm{~mm}$ & No Activity \\
\hline 21 & $X G$ & Shigellaflexneri & No Activity & No Activity & No Activity & $40 \mathrm{~mm}$ & No Activity \\
\hline 22 & $X G$ & Sphingomonaspaucimobilis & No Activity & No Activity & No Activity & $31 \mathrm{~mm}$ & No Activity \\
\hline 23 & $X G$ & Escherichia coli & $12 \mathrm{~mm}$ & $11 \mathrm{~mm}$ & No Activity & $40 \mathrm{~mm}$ & No Activity \\
\hline 24 & $X G$ & Vibrio cholerae & No Activity & No Activity & No Activity & $40 \mathrm{~mm}$ & No Activity \\
\hline
\end{tabular}

Diameter of the well $=06 \mathrm{~mm}$

Volume of the Compound = 50 Micro liters. 


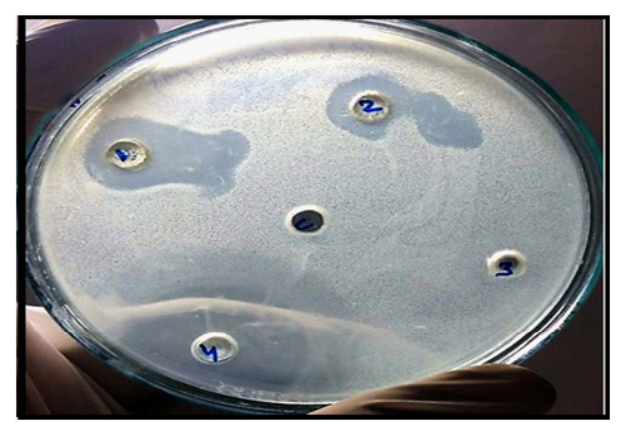

Fig. 3: Mangrove plants extracts and a new flavone compound activity on some selected strains

are Bacillus subtilis with the values 19,18 and 17 respectively. These strains are no activity was found against Bacillus subtilis and Bacillus coagulans. Staphylococcus aureus with the values 13, 12 and 11 respectively. Bacilluslicheniformis with the values 14,12 and 11 respectively.Corynebacterium diphtheria with the values 11 and 10 respectively. The Gram Negative Bacteria's are Klebsiella pneumonia and Shigella flexneri with the value 11 respectively. And Pseudomonas aeruginosa with the value 10 respectively. And Vibrio cholera\&Sphingomonas paucimobilis with the values $13 \& 15$ respectively.

With RM extract, the antimicrobial activity for strains

Bacillus puvuilis, Bacillus subtilis, Bacillus coagulans, Staphylococcus aureus, Bacillus licheniformis, Corynebacterium diphtheria, Klebsiella pneumonia, Pseudomonas aeruginosa, Shigella flexneri, Sphingomonas paucimobilis, Escherichia coli and Vibrio cholera.T he Gram Positive Bacteria's are Bacillus subtilis \& Bacillus coagulans with the values $12 \& 15,13,11$ respectely. These strains have no activity was found against Bacillus subtilis, Staphylococcus aureus, Bacillus licheniformis and Corynebacteriumdiphtheria.

The Gram Negative Bacteri's Klebsiella pneumonia with the values 20,13 and 10 respectively. And Pseudomonas aeruginosa with the value 15 respectively, Shigella flexneri with the values 16 and 12 respectively, Sphingomonaspaucimobilis with the values 19, 13 and 11 respectively. Escherichia coli with the values 16 and 12 respectively. And Vibrio cholera with the values 19,13 and 10 respectively.

\section{With XG extract, the antimicrobial activity for} strains

Bacillus puvuilis, Bacillus subtilis, Bacillus coagulans, Staphylococcus aureus, Bacillus licheniformis, Corynebacterium diphtheria, Klebsiella pneumonia, Pseudomonas aeruginosa, Shigella flexneri, Sphingomonas paucimobilis, Escherichia coli and Vibrio cholera. The Gram Positive Bacteria's are Bacillus puvuilis \& Corynebacterium diphtheria these strains are no activity. And Bacillus subtilis with the values 12 and 10 respectively, Bacillus coagulans with the values 15 and 11 respectively, Staphylococcus aureus with the values 14, 13 and 12 etc.And Bacillus licheniformis with the values 11 and 10 respectively.The Gram Negative Bacteria's are Escherichia coli with the values 12 and 11 respectively. Remaining in all Negative Strains has no activity.

Finally the order of effectiveness is found to be: EA Hexane > RM MeOH > XG MeOH > AG $\mathrm{MeOH}$. Finally a new flavone compound is found that more effective than the extracts.

The order of Activity is:EA Hexane (4) > $\mathrm{RM} \mathrm{MeOH} \mathrm{(1)} \mathrm{>} \mathrm{XG} \mathrm{MeOH} \mathrm{(2)>} \mathrm{AGMeOH} \mathrm{(3).}$

\section{CONCLUSION}

The extracts and new flavone compound of parts of different species of mangrove plants have been tested for their antimicrobial activity towards the strains Bacillus puvuilis, Bacillus subtilis, Bacillus coagulans, Staphylococcus aureus, Bacillus licheniformis, Corynebacterium diphtheria, Klebsiella pneumonia, Pseudomonas aeruginosa, Shigella flexneri, Sphingomonas paucimobilis, Escherichia coli and Vibrio cholera adopting Agarwell diffusion method. It is found that EA Hexane Flavone Compound is effective towards Bacillus puvuilis, Bacillus subtilis, Bacillus coagulans, Staphylococcus aureus, Bacillus licheniformis, Corynebacterium diphtheria, Klebsiella pneumonia, Pseudomonas aeruginosa, Shigella flexneri, Sphingomonas paucimobilis, Escherichia coli and Vibrio cholera strains while $\mathrm{RM} \mathrm{MeOH}$ extract is effective towards the strains Bacillus puvuilis, Bacillus subtilis, Bacillus coagulans, Staphylococcus aureus, Bacillus licheniformis, Corynebacterium diphtheria, Klebsiella pneumonia, Pseudomonas 
aeruginosa, Shigella flexneri, Sphingomonas paucimobilis, Escherichia coli and Vibrio cholera. The $\mathrm{XG} \mathrm{MeOH}$ extract is found to be effective towards the strains Bacillus puvuilis, Bacillus subtilis, Bacillus coagulans, Staphylococcus aureus, Bacillus licheniformis, Corynebacterium diphtheria, Klebsiella pneumonia, Pseudomonas aeruginosa, Shigella flexneri, Sphingomonas paucimobilis, Escherichia coli and Vibrio cholera strains while AGC MeOH extract is found to be effective towards the strains Bacillus puvuilis, Bacillus subtilis, Bacillus coagulans, Staphylococcus aureus, Bacillus licheniformis, Corynebacterium diphtheria, Klebsiella pneumonia, Pseudomonas aeruginosa,
Shigella flexneri, Sphingomonas paucimobilis, Escherichia coli and Vibrio cholera. The order of effectiveness is found to be: EA Hexane > RM $\mathrm{MeOH}>\mathrm{XG} \mathrm{MeOH}>\mathrm{AG} \mathrm{MeOH}$. Finally a new flavone compound is found that more effective than the extracts.

\section{ACKNOWLEDGEMENT}

We are thankful to Department of Chemistry K L University, Andhra pradesh, India for making available the necessary facilities. We are grateful to Dr.Varaprasad Bobbarala ADHYA BIOSCIENCE PVT.LTD, Visakhapatnam for his suggestions.

\section{REFERENCES}

1. Ji-Dong W.; Wen Z.; Zhen-Y L.; Wen-Sheng X.; Yue-Wei G. and Krohn K. Phytochemistry. 2007, 68: 24-26.

2. Wang J.D.; Li Z Y.; Xiang W.S.; Guo Y.W. Helvetica Chimica Acta. 2006, 89: 13671375.

3. Ericson K.L.; Beutler J.A.; Cardellina J.H.; McMahon J.B.; Newman J.D.; Boyd M.R. Journal of Natural Products. 1995, 58: 769775.

4. Pritinanda M.;Suman J.; Santilata S. Int. Journal of Science, Technology \& Management.2015, 4: 2394-2399.

5. Madhurima.B.; Punarbasu.C. Int.Journal of Pharma and Bio Sciences.2014, 5: 294-304.

6. Pandey R.; Pandey C.N. Journal of Plant Studies. 2013, 2: 53-60.

7. Jun Wu.; Haixin Ding.;Minyi. Li.; Si Zhang.
Z.Naturforsch. 2007, 62b: 569.

8. Yuan Zhou.; Jun Wu.; Kun Zou. Chemistry of Natural Compounds. 2007, 43: 426-432.

Khisal A. Alvi.; Phil Crews.; Bill Aalbersberg.; Regina Prasad. Tetrahedron.1991, 47: 8943.

10. Elsa Lycias. Joel.; Valentin. Bhimba., Asian Pacific Journal of Tropical Medicine, 2010, 602.

11. Powar P. S.; Gaikwad D.K. Int Journal of Pharma \& Bio Science.2013, 4: 141-155.

12. Gurudeeban.S.; Ramanathan. Pharmaceutical Chemistry Journal. 2013, 47.

13. Rajeswari.K.;BhaskaraRao.T.; G.V.R.Sharma.; MuraliKrishna.R. Der PharmaChemica. 2016, 8: 509-514.

14. Qaralleh H.; Did S.;Saad S.;Susanti D.;Taher M.;Khleifat K., J Med Mycol,2010, 20: 315320. 